\title{
Drug allergy
}

\author{
Richard Warrington ${ }^{1 *}$, Fanny Silviu-Dan ${ }^{2}$ and Tiffany Wong ${ }^{3}$
}

\begin{abstract}
Drug allergy encompasses a spectrum of immunologically-mediated hypersensitivity reactions with varying mechanisms and clinical presentations. This type of adverse drug reaction not only affects patient quality of life, but may also lead to delayed treatment, unnecessary investigations, and even mortality. Given the myriad of symptoms associated with the condition, diagnosis is often challenging. Therefore, referral to an allergist experienced in the identification, diagnosis and management of drug allergy is recommended if a drug-induced allergic reaction is suspected. Diagnosis relies on a careful history and physical examination and, in some instances, skin testing and graded challenges. Induction of drug tolerance procedures may also be required. The most effective strategy for the management of drug allergy is avoidance or discontinuation of the offending drug. When available, alternative medications with unrelated chemical structures should be substituted. Cross-reactivity among drugs should be taken into consideration when choosing alternative agents. Additional therapy for drug hypersensitivity reactions is largely supportive and may include topical corticosteroids, oral antihistamines and, in severe cases, systemic corticosteroids. In the event of anaphylaxis, the treatment of choice is injectable epinephrine. If a particular drug to which the patient is allergic is indicated and there is no suitable alternative, induction of drug tolerance procedures may be considered to induce temporary tolerance to the drug. This article provides a background on drug allergy and strategies for the diagnosis and management of some of the most common drug-induced allergic reactions, such as penicillin, sulfonamides, cephalosporins, radiocontrast media, local anesthetics, general anesthetics, acetylsalicylic acid and nonsteroidal anti-inflammatory drugs, and therapeutic monoclonal antibodies.
\end{abstract}

\section{Background}

An adverse drug reaction (ADR) is defined as any harmful or unintended reaction to a drug that occurs at doses used for prevention, diagnosis, or treatment [1]. ADRs are common in everyday clinical practice, affecting between 15 and $25 \%$ of patients; serious reactions occur in $7-13 \%$ of patients [2, 3].

ADRs are classified as either predictable reactions that may occur in anyone (type A) or unpredictable reactions that occur in susceptible individuals (type B) (Table 1). Predictable reactions are the most common type of ADR and are usually dose dependent and related to the known pharmacologic actions of the drug (e.g., side effects, overdose, drug interactions). Unpredictable reactions occur in approximately $20-25 \%$ of patients who experience ADRs; these reactions are generally unrelated to the pharmacologic actions of the drug $[1,4,5]$.

\footnotetext{
*Correspondence: richard.warrington@gmail.com

${ }^{1}$ University of Manitoba, Winnipeg, MB, Canada

Full list of author information is available at the end of the article
}

Drug allergy is one type of unpredictable ADR that encompasses a spectrum of immunologically-mediated hypersensitivity reactions with varying mechanisms and clinical presentations [1]. It accounts for approximately $5-10 \%$ of all ADRs [6]. Pseudoallergic reactions (also known as non-allergic or non-immune-mediated reactions) represent another type of unpredictable ADR. These reactions are often clinically indistinguishable from true immunologically mediated allergic reactions, but they lack immunological specificity.

Drug allergy not only affects patient quality of life, but may also lead to delayed treatment, use of suboptimal alternate medications, unnecessary investigations, increased morbidity and even death. Furthermore, identification of drug allergy is challenging given the myriad of symptoms and clinical presentations associated with the condition. Therefore, if a druginduced allergic disorder is suspected, consultation with an allergist experienced in the identification, diagnosis and management of drug allergy is recommended. This 
Table 1 Classification of adverse drug reactions $[1,4,5]$

\begin{tabular}{l|l}
\hline Type A: predictable & Type B: unpredictable \\
\hline -Drug overdose & $\begin{array}{l}\text {-Drug allergy: an immunologically mediated ADR } \\
\text { - Secondary drug effects } \\
\text { - Side effects } \\
\text {-Drug interactions } \\
\text { immunological specificity } \\
\text {-Drug intolerance: an undesirable pharmacologic effect that occurs at low and sometimes sub-therapeutic doses of the } \\
\text { drug that are not caused by underlying abnormalities of metabolism or drug excretion } \\
\text {-Drug idiosyncrasy: an abnormal/unexpected effect, usually caused by underlying abnormalities of metabolism, excretion, } \\
\text { or bioavailability }\end{array}$ \\
\hline
\end{tabular}

$A D R$ adverse drug reaction

article will provide an overview of the mechanisms and risk factors for drug allergy, as well as strategies for the diagnosis and appropriate management of some of the most common drug-induced allergic disorders.

\section{Mechanisms}

Immune-mediated allergic reactions to drugs are classified according to the Gell and Coombs' classification system, which describes the predominant immune mechanisms involved in these reactions. This classification system includes: immediate-type reactions mediated by immunoglobulin $\mathrm{E}$ (IgE) antibodies (type I), cytotoxic reactions mediated by immunoglobulin $\mathrm{G}$ (IgG) or immunoglobulin M (IgM) antibodies (type II), immune-complex reactions (type III), and delayed-type hypersensitivity reactions mediated by cellular immune mechanisms, such as the recruitment and activation of T cells (type IV) [7-9]. The mechanisms, clinical manifestations, and timing of these immune reactions are summarized in Table 2.

There are two theories to explain how a low molecular weight compound such as a drug is able to stimulate an immune response: (1) the hapten hypothesis and (2) the pharmacological-interaction (p-i) hypothesis [10]. In the hapten theory, the drug binds to a macromolecule such as a serum protein like albumin, which is then processed by antigen-presenting cells (APCs) and presented to $\mathrm{T}$ cells that recognize the modified self protein. The $\mathrm{p}-\mathrm{i}$ hypothesis proposes that the drug binds to a surface receptor, such as the major histocompatibility complex (MHC) or the T cell receptor, and modifies its structure so that it is recognized by other cells of the adaptive immune system as foreign, thereby stimulating an immune response.

For high molecular weight therapeutic agents such as monoclonal antibodies (mAbs), these often contain murine-derived structures which are recognized as foreign by the immune system, resulting in primarily type I (IgE-mediated) or type III (immune-complex-mediated) reactions.

Unlike immune-mediated drug reactions, pseudoallergic reactions are not associated with the production of antibodies or sensitized $\mathrm{T}$ cells, but are often clinically indistinguishable from drug

Table 2 Classification of allergic drug reactions: mechanisms, clinical manifestations, and timing of reactions [6-9].

\begin{tabular}{|c|c|c|c|}
\hline Immune reaction & Mechanism & Clinical manifestations & Timing of reaction \\
\hline Type I (lgE-mediated) & $\begin{array}{l}\text { Drug-lgE complex binding to mast } \\
\text { cells with release of histamine, } \\
\text { inflammatory mediators }\end{array}$ & $\begin{array}{l}\text { Anaphylaxis }{ }^{a}, \text { urticaria }^{a} \text {, angioedema } \\
\text { bronchospasm }\end{array}$ & Minutes to hours after drug exposure \\
\hline Type II (cytotoxic) & $\begin{array}{l}\text { Specific lgG or lgM antibodies } \\
\text { directed at drug-hapten coated } \\
\text { cells }\end{array}$ & $\begin{array}{l}\text { Anemia, cytopenia, } \\
\text { thrombocytopenia }\end{array}$ & Variable \\
\hline Type III (immune complex) & $\begin{array}{l}\text { Tissue deposition of drug-antibody } \\
\text { complexes with complement } \\
\text { activation and inflammation }\end{array}$ & $\begin{array}{l}\text { Serum sickness, vasculitis, fever, rash, } \\
\text { arthralgia }\end{array}$ & 1-3 weeks after drug exposure \\
\hline Type IV (delayed, cell mediated) & $\begin{array}{l}\text { MHC presentation of drug molecules } \\
\text { to T cells with cytokine and } \\
\text { inflammatory mediator release; may } \\
\text { also be associated with activation } \\
\text { and recruitment of eosinophils, } \\
\text { monocytes, and neutrophils }\end{array}$ & $\begin{array}{l}\text { Contact sensitivity, skin rashes, organ- } \\
\text { tissue damage }\end{array}$ & 2-7 days after drug exposure \\
\hline
\end{tabular}

Adapted from Riedl et al. [6]

IgE immunoglobulin E, IgG immunoglobulin G, IgM immunoglobulin G, MHC major histocompatibility complex

a These reactions may also be non-immunologically mediated 
hypersensitivity reactions. During these reactions, the drug has the ability, via its chemistry or pharmacology, to directly stimulate the release or activation of inflammatory mediators such as histamine (from mast cells, basophils), prostaglandins, leukotrienes, or kinins. Non-steroidal anti-inflammatory drugs (NSAIDs), opioids, and angiotensin-converting enzyme (ACE) inhibitors are common causes of these non-allergic reactions $[5,11,12]$.

\section{Risk factors}

Factors associated with an increased risk of developing a drug allergy include patient-related factors (e.g., age, gender, genetic polymorphisms, or infections with certain viruses) and drug-related factors (e.g., frequency of exposure, route of administration, or molecular weight) (Table 3). Drug allergy typically occurs in young and middle-aged adults, and is more common in women. Genetic polymorphisms in the human leukocyte antigen (HLA; a gene product of the MHC) as well as viral infections such as human immunodeficiency virus (HIV) and the Epstein-Barr virus (EBV), have also been linked to an increased risk of developing immunologic reactions to drugs. Susceptibility to drug allergy is influenced by genetic polymorphisms in drug metabolism. In addition, topical, intramuscular, and intravenous (IV) routes of administration are more likely to cause allergic drug reactions than oral administration. IV administration is associated with more severe reactions. Prolonged high doses or frequent doses of medication are more likely to lead to hypersensitivity reactions than a large single dose. Furthermore, large macromolecular drugs (e.g., insulin or horse antisera) or drugs that haptenate (bind to tissue or blood proteins and elicit an immune response), such as penicillin, are also associated with a greater likelihood

Table 3 Risk factors for the development of drug allergy [16]

Patient-related factors
-Age: young/middle-aged adults > infants/elderly
-Gender: Women > men
-Genetic polymorphisms
-Viral infections: HIV, herpes viruses
-Previous reaction to the drug
Drug-related factors
-High molecular weight compounds and hapten-forming drugs are
more immunogenic
-Route: topical >IV/intramuscular $>$ oral
-Dose: frequent/prolonged $>$ single dose

HIV human immunodeficiency virus of causing hypersensitivity reactions. Although atopic patients do not have an increased risk for drug allergy, they are at increased risk for serious allergic reactions [4, $6,13-16]$.

\section{Diagnosis}

The diagnosis of drug allergy requires a thorough history and the identification of physical findings and symptoms that are compatible with the characteristics and timing of drug-induced allergic reactions. Depending on the history and physical examination, diagnostic tests such as skin testing and graded challenges may be required $[1,4$, $6,16]$. Therefore, if drug allergy is suspected, evaluation by an allergist experienced in these diagnostic procedures is recommended.

\section{History}

Evaluation of the patient with a suspected drug allergy should include a detailed history of all prescription and nonprescription drugs taken by the patient, including dates of administration, drug formulation, dosage and route of administration, clinical symptoms and their timing and duration in relation to drug exposure, as well as previous and subsequent drug exposures and reactions $[1,4,6,16]$.

\section{Clinical presentation}

In addition to the detailed history, a careful physical examination can help to define possible mechanisms underlying the reaction and guide subsequent investigations and diagnostic testing. Table 4 highlights some of the most common clinical manifestations of drug allergy and examples of causative drugs.

The skin is the organ most frequently and prominently affected by drug-induced allergic reactions $[1,6,12]$. The most common cutaneous manifestation is a generalized maculopapular rash, which is characterized by raised, pink or erythematous lesions that appear within days to 3 weeks after drug exposure. Lesions typically originate in the truncal area and eventually spread to the limbs. Urticaria (hives) and angioedema (swelling) are also common, and can result from both IgE-mediated and non-IgE-mediated mechanisms. Compared with the adult population, the most likely cause of delayed maculopapular rashes and acute urticaria/angioedema in the pediatric population is a viral infection, and children with these presentations have a lower rate of drug allergy $[17,18]$. The most severe forms of cutaneous drug reactions are Stevens-Johnson syndrome (SJS) and toxic epidermal necrolysis (TEN). SJS begins with a maculopapular rash that often progresses to bullae, mucous membrane ulcerations, conjunctivitis, fever, sore throat and fatigue. TEN is a rare condition with similar 
Table 4 Clinical manifestations of drug allergy $[1,12,16]$

\begin{tabular}{|c|c|c|}
\hline Manifestation & Clinical features & Examples of causative drugs \\
\hline \multicolumn{3}{|l|}{ Skin } \\
\hline Exanthemata & $\begin{array}{l}\text {-Diffuse, fine macules and papules } \\
\text {-Evolve over days post drug initiation }\end{array}$ & $\begin{array}{l}\text { Allopurinol, penicillins, cephalosporins, anticonvulsants, } \\
\text { sulfonamides, mAbs }\end{array}$ \\
\hline Urticaria, angioedema & $\begin{array}{l}\text { - Onset within minutes to hours of drug administration } \\
\text {-Potential for anaphylaxis } \\
\text { - Often lgE-mediated }\end{array}$ & $\begin{array}{l}\text { Antibiotics, ACE inhibitors, anticonvulsants, neuromuscular } \\
\text { blocking agents, platinums, radiocontrast media, NSAIDs, } \\
\text { narcotics, mAbs }\end{array}$ \\
\hline Fixed drug eruption & $\begin{array}{l}\text {-Hyper-pigmented plaques that occur at the same site upon } \\
\text { re-exposure to the culprit drug }\end{array}$ & $\begin{array}{l}\text { Sulfonamide and tetracycline antibiotics, NSAIDs, ASA, } \\
\text { sedatives, chemotherapeutic agents, anticonvulsants }\end{array}$ \\
\hline SJS & $\begin{array}{l}\text { - Fever, sore throat, fatigue, ocular involvement } \\
\text { - Ulcers and other lesions on mucous membranes, particularly } \\
\text { of the mouth and lips, as well as on truncal area }\end{array}$ & $\begin{array}{l}\text { Sulfonamides, nevirapine, corticosteroids, anticonvulsants, } \\
\text { NSAIDs (oxicams), allopurinol, phenytoin, carbamazepine, } \\
\text { lamotrigine, barbiturates, psychotropic agents, } \\
\text { pantoprazole, tramadol, mAbs }\end{array}$ \\
\hline TEN & $\begin{array}{l}\text {-Similar to SJS, but usually involves significant epidermal } \\
\text { detachment } \\
\text { •Potentially life-threatening }\end{array}$ & Same as SJS \\
\hline Hematologic & •Hemolytic anemia, leukopenia, thrombocytopenia & $\begin{array}{l}\text { Penicillin, sulfonamides, anticonvulsants, cephalosporins, } \\
\text { quinine, heparin, thiazides, gold salts }\end{array}$ \\
\hline Hepatic & -Hepatitis, cholestatic jaundice & $\begin{array}{l}\text { Sulfonamides, phenothiazines, carbamazepine, erythromycin, } \\
\text { anti-tuberculosis agents, allopurinol, gold }\end{array}$ \\
\hline Renal & -Interstitial nephritis, glomerulonephritis & $\begin{array}{l}\text { Penicillin, sulfonamides, allopurinol, PPIs, ACE inhibitors, } \\
\text { NSAIDs }\end{array}$ \\
\hline \multicolumn{3}{|l|}{ Multi-organ reactions } \\
\hline Anaphylaxis & $\begin{array}{l}\text {-Urticaria/angioedema, bronchospasm, gastrointestinal } \\
\text { symptoms, hypotension }\end{array}$ & $\begin{array}{l}\text { Antibiotics, neuromuscular blocking agents, anesthetics, } \\
\text { radiocontrast media, recombinant proteins (e.g., } \\
\text { omalizumab) }\end{array}$ \\
\hline DRESS & $\begin{array}{l}\text {-Cutaneous eruption, fever, eosinophilia, hepatic dysfunction, } \\
\text { lymphadenopathy }\end{array}$ & $\begin{array}{l}\text { Anticonvulsants, sulfonamides, minocycline, allopurinol, } \\
\text { strontium ranelate, mAbs }\end{array}$ \\
\hline Serum sickness & -Urticaria, arthralgias, fever & $\begin{array}{l}\text { Heterologous antibodies, infliximab, allopurinol, thiazides, } \\
\text { antibiotics (e.g., cefaclor), bupropion, mAbs }\end{array}$ \\
\hline DILE & -Arthralgias, myalgias, fever, malaise & $\begin{array}{l}\text { Hydralazine, procainamide, isoniazid, quinidine, minocycline, } \\
\text { antibiotics, and anti-TNF-alpha agents }\end{array}$ \\
\hline Vasculitis & -Cutaneous or visceral vasculitis & $\begin{array}{l}\text { Sulfonamide antibiotics and diuretics, hydralazine, } \\
\text { penicillamine, propylthiouracil, mAbs }\end{array}$ \\
\hline
\end{tabular}

ACE angiotensin-converting enzyme, NSAIDs non-steroid anti-inflammatory drugs, SJS Stevens-Johnson syndrome, TEN toxic epidermal necrolysis, DRESS drug rash with eosinophilia and systemic symptoms, DILE drug-induced lupus erythematosus, ASA acetylsalicylic acid, PPIs proton pump inhibitors, TNF tumour necrosis factor, mAbs monoclonal antibodies

characteristics to SJS, but it also causes large portions of the epidermis (the skin's outermost layer) to detach from the layers below, leading to extensive skin exfoliation and a scalded skin appearance. Given the severity of these conditions, drugs suspected of causing SJS and TEN (most commonly sulfonamides) should be strictly avoided by the patient in the future [1].

Although skin reactions are the most common physical manifestation of drug-induced allergic reactions, many other organ systems may be involved, such as the renal, hepatic and hematologic systems (Table 4). Multi-organ reactions may also occur and include anaphylaxis (see Anaphylaxis article in this supplement), drug rash with eosinophilia and systemic symptoms (DRESS) syndrome, serum sickness, drug-induced lupus erythematosus (DILE) and vasculitis (a heterogeneous group of disorders that are characterized by inflammatory destruction of blood vessels).
DRESS is a potentially life-threatening condition characterized by a widespread rash, fever, lymphadenopathy (swollen/enlarged lymph nodes) and hepatic dysfunction. Serum sickness is an immunecomplex-mediated reaction that presents with fever, lymphadenopathy, arthralgia, and cutaneous lesions.

Classic serum sickness is caused by heterologous proteins, such as rabbit antithymocyte globulin or equine-derived anti-toxins, and is more common in adults. Serum sickness-like reactions are more common in children and tend to occur after infections or administration of some vaccines or drugs such as cefaclor and penicillin. However, serum sickness-like reactions may also occur with newer mAbs that contain foreign murine components in the variable regions. The exact mechanism of serum sickness-like reactions is poorly understood. 
The typical symptoms of DILE include sudden onset of fever and malaise; myalgia, arthralgia, and arthritis may also occur several weeks after drug initiation. In approximately $25 \%$ of cases, the skin may also be affected $[1,12]$.

Serum sickness and DILE are usually self-limited, with symptoms resolving spontaneously within a few weeks after discontinuation of the offending drug. However, the symptoms of DRESS may worsen or persist for weeks, or even months, following drug discontinuation $[1,12]$.

Since the clinical manifestations of drug allergy are highly variable, it is important to exclude other conditions that may mimic drug-induced allergic reactions. Table 5 lists some of the conditions that should be considered in the differential diagnosis of drug allergy.

\section{Diagnostic tests}

Skin testing procedures, such as skin prick testing (SPT) and intradermal tests (test in which the allergen is injected into the skin dermis) are useful for the diagnosis of IgE-mediated (type I) reactions. Skin testing protocols are standardized for penicillin, and are also useful (but rarely positive) for local anesthetics, muscle relaxants, and very sensitive for high-molecular-weight protein substances, such as insulin or mAbs. Positive skin tests to these drugs confirm the presence of antigen-specific $\mathrm{IgE}$ and support the diagnosis of a type I hypersensitivity reaction. The negative predictive value of penicillin skin testing is high with appropriate reagents and, therefore, a negative test result is useful for ruling out penicillin allergy. With other agents (except high molecular weight proteins), however, a negative skin test does not effectively rule out the presence of specific IgE. Serumspecific IgE tests are available for a limited number of drugs. However, these tests are costly and generally less sensitive than skin tests. Furthermore, most of these in vitro tests are not adequately validated for drug allergy testing $[1,16]$. Therefore, in most clinical settings, serumspecific IgE tests for medications are not used for the diagnosis of drug allergy.
Patch testing involves placing potential allergens (at non-irritant concentrations) on the patient's back for $48 \mathrm{~h}$ under aluminum discs, and then assessing for reactions. Drug patch testing is useful for the diagnosis of various delayed (type IV) cutaneous reactions, particularly exanthemata, but is generally not helpful for the diagnosis of SJS or TEN $[1,11,12,16,19]$.

The measurement of histamine and tryptase levels have proved useful in confirming acute IgE-mediated reactions, particularly anaphylaxis; however, negative results do not rule out acute allergic reactions. A complete blood count can help diagnose hemolytic (type II) drug-induced reactions, such as hemolytic anemia, thrombocytopenia, or neutropenia. Hemolytic anemia may also be confirmed with a positive direct and/or indirect Coombs' test (used to examine for the presence of antibodies on red blood cell membranes) $[1,12,16]$.

Recent studies have focused on the potential role of the basophil activation test (the quantification of basophil activation by flow cytometry) in the diagnosis of drug allergy, since basophils are involved in both immune-mediated and non-immune-mediated reactions. Although some evidence suggests that the test is useful for evaluating possible allergies to beta-lactam antibiotics, NSAIDs and muscle relaxants, further confirmatory studies are needed before it is widely accepted as a diagnostic tool $[1,20,21]$.

In cases where there is a definite medical need for a particular drug, but the clinical diagnosis of drug allergy remains uncertain despite thorough investigations, a procedure to induce temporary drug tolerance (also referred to as drug desensitization) or graded challenge testing (also known as provocation testing) may be considered. Induction of drug tolerance procedures temporarily modify a patient's immunologic or non-immunologic response to a drug through the administration of incremental doses of the drug. Most regimens begin with a very dilute concentration of the drug, and the dose is doubled every 15-20 min, until a full therapeutic dose has been administered after 3-8 $\mathrm{h}$.

Table 5 Conditions to consider in the differential diagnosis of drug allergy [5]

\begin{tabular}{l|l}
\hline IgE-mediated drug allergy (urticaria, angioedema, anaphylaxis, bronchospasm) & Non-lgE mediated reactions (exanthema, DRESS, SJS, TEN) \\
\hline -Carcinoid syndrome & -Acute graft-versus-host disease \\
-Insect bites/stings & -Kawasaki disease \\
-Mastocytosis & -Still's disease \\
-Asthma & -Psoriasis \\
- Food allergy & -Insect bites/stings \\
-Scombroid fish poisoning & -Viral infection \\
- Latex allergy & -Streptococcal infection \\
-Infection (EBV, hepatitis A, B, C, gastrointestinal parasites) & \\
\hline
\end{tabular}

IgE immunoglobulin E, EBV Epstein-Barr virus, SJS Stevens-Johnson syndrome, TEN toxic epidermal necrolysis, DRESS drug rash with eosinophilia and systemic symptoms 
Drug tolerance is usually maintained only as long as the drug is administered; the procedure needs to be repeated in the future if the patient requires the drug again after finishing a prior therapeutic course. Unlike induction of drug tolerance procedures, graded challenge tests do not modify a patient's immunologic or non-immunologic response to a given drug. These tests are generally used to determine whether a patient will have an adverse reaction to a particular drug by administering sub-therapeutic doses over a period of time, while observing the patient for potential reactions. Graded challenge tests involve a maximum of 3-4 doses; using more doses can result in induction of drug tolerance and an erroneous impression that a patient will tolerate the medication on subsequent exposures. They are not advised if the patient has experienced a previous life-threatening reaction to the drug in question. Drug tolerance-induction procedures and graded challenges are potentially harmful and should only be performed by experienced personnel in facilities with resuscitative equipment readily available $[1,22]$.

\section{Management of common drug allergies}

The most effective strategy for the management of drug allergy is avoidance or discontinuation of the offending drug. When available, alternative medications with unrelated chemical structures should be substituted. Cross-reactivity among drugs should be taken into consideration when choosing alternative agents $[1,12]$.

Additional therapy for drug hypersensitivity reactions is largely supportive and symptomatic. For example, topical corticosteroids and oral antihistamines may improve cutaneous symptoms. In the event of anaphylaxis, the treatment of choice is epinephrine administered by intramuscular injection into the lateral thigh. Systemic corticosteroids may also be used to treat severe systemic reactions, but should never be given prior to or replace epinephrine in the treatment of anaphylaxis. Severe drug reactions, such as SJS and TEN, are best treated in an intensive care or burn unit setting [1,12]. Strategies for the management of some of the most common drug allergies are discussed below.

\section{Penicillin}

Penicillin is the most frequent drug allergy, affecting approximately $10 \%$ of patients. For patients with penicillin allergy, treatment is best limited to nonpenicillin agents. Carbapenems (e.g., imipenem) do not exhibit a significant degree of cross-reactivity with penicillin and may be administered as a graded challenge after prophylactic skin tests with the relevant carbapenem [23, 24]. Monobactams, such as aztreonam, are generally well tolerated by patients with penicillin allergy, except if they had an allergic reaction to ceftazidime [25-27]. Second- or third-generation cephalosporins may also be considered since the degree of cross reactivity with these agents and penicillin has been shown to be lower than with first-generation agents (see following "Cephalosporin" section) $[1,28]$.

Ideally, management of the patient with penicillin allergy should include penicillin skin testing. Approximately $90 \%$ of patients have negative penicillin skin test responses and can safely receive cephalosporins as well as other beta-lactam agents. If a penicillin is deemed absolutely necessary in a penicillin-allergic patient, desensitization should be considered, and the procedure should only be performed under medical supervision in-hospital [1].

\section{Cephalosporins}

The most common allergic reactions to cephalosporins are maculopapular rashes and drug fever; urticaria is less common and anaphylaxis is rare [28]. As mentioned earlier, positive skin tests to penicillin are associated with a higher likelihood of allergic reactions to first-generation cephalosporins (about 2\%) [29]. However, this cross reactivity was based on skin testing and not challenge testing. In contrast, Macy and Ngor found the incidence of clinical reactions to first-generation cephalosporins to be the same as to sulphonamides in pencillin-intolerant patients [30]. In pencillin-allergic patients, it may be advisable to avoid first-generation cephalosporins unless skin testing to an appropriate cephalosporin is negative. In cephalosporin-allergic subjects, there is limited cross reactivity on immunological testing between secondand third-generation cephalosporins and penicillins, especially amino-penicillins, but this has not necessarily indicated clinical reactivity [31]. There is a role for skin testing with the proposed antibiotic to be used in therapy, and/or administration by graded challenge. If skin testing is positive and no alternative drug exists, induction of drug tolerance procedures may be attempted $[1,5]$.

\section{Sulfonamides}

Sulfonamide antibiotics are another common cause of drug-induced allergic reactions, and are often associated with delayed cutaneous maculopapular eruptions, SJS and TEN. Patients infected with HIV are at increased risk of developing cutaneous reactions to sulfonamide antibiotics, which is likely related to immunologic factors and frequent exposure to these antibiotics. Trimethoprim-sulfamethoxazole (TMP-SMX) is the drug of choice for the prophylaxis and treatment of a number of opportunistic infections and, therefore, many HIV-positive patients with a history of reacting to sulfonamides still require treatment with this antibiotic. Induction of drug tolerance procedures can be used to 
safely administer TMP-SMX to HIV-positive patients with a history of reacting to the antibiotic.

Since the chemical structure of non-antibiotic sulfonamides (e.g., thiazide diuretics, some NSAIDs and anticonvulsants) varies from sulfonamide antibiotics, these agents are not expected to cross-react, and can generally be safely administered to patients with a history of allergy to sulfonamide antibiotics. An exception is sulfasalazine which, by intestinal degradation becomes sulfapyridine, acquiring an aromatic immunogenic structure like sulfamethoxazole [1, 32-34].

\section{Radiocontrast media}

Radiocontrast media (RCM) are associated with both allergic and pseudoallergic reactions. The incidence of reactions to RCM, including severe, life-threatening reactions, appears to be lower with non-ionic versus ionic agents. Pseudo/allergic reactions to RCM can usually be prevented through the use of pretreatment regimens that include oral corticosteroids and H1-antihistamines. Low osmolarity agents should also be used in such circumstances $[1,5]$.

\section{Local anesthetics}

True allergic reactions to local anesthetics (e.g., novocaine, lidocaine) are extremely rare; reactions are usually due to other ingredients in the medication, such as preservatives or epinephrine. However, if the reaction history is consistent with a possible immediate, IgE-mediated (type I) reaction, skin testing followed by graded challenge tests using epinephrine-free, preservative-free local anesthetics may be utilized [1].

\section{General anesthetics}

Although rare, anaphylaxis may occur in patients under general anesthesia. The investigation of severe reactions during general anesthesia is particularly challenging given that the patient is often exposed to many co-administered drugs and agents. Reactions during general anesthesia are often due to neuromuscular blocking agents and antibiotics [35], but have also been associated with IV anesthetics (e.g., propofol, thiopentone, etomidate), NSAIDs, chlorhexidine, and latex allergy. Also, opioids may be confounders as they can either mimic or amplify these reactions. There are no reported cases of allergy to inhaled anesthetics. Assessment by an allergist is important for confirming the clinical diagnosis of allergy to general anesthesia, identifying likely causative agents as well as alternative agents that may be used safely in the future [36].

\section{Acetylsalicylic acid/NSAID reactions}

Acetylsalicylic acid (ASA) and NSAIDs can cause both true allergic and pseudoallergic reactions, including exacerbations of underlying respiratory diseases, urticaria, angioedema, and anaphylaxis. Patients with underlying chronic respiratory diseases, such as asthma, rhinitis, and sinusitis, may react to ASA and NSAIDs that inhibit cyclooxygenase-1 (COX-1). The management of these patients involves avoidance of aspirin and NSAIDs and aggressive treatment of the underlying respiratory disorder. Selective COX-2 inhibitors almost never cause reactions, and can typically be taken safely by patients with ASA/NSAID allergy. An induction of drug tolerance procedure to aspirin (also known as aspirin desensitization) may also be considered in aspirinexacerbated respiratory diseases [1].

Patients with chronic urticaria/angioedema generally tolerate COX-2 inhibitors, but may experience exacerbations of urticaria/angioedema with NSAIDs that inhibit COX-1. True allergic reactions to NSAIDs are usually drug specific and, therefore, patients experiencing these reactions are often able to tolerate other NSAIDS [1].

\section{Monoclonal antibody (mAb) reactions}

mAbs are proteins with inherent immunogenicity. Hypersensitivity reactions to mAbs, which can range in severity from mild to life-threatening, represent an escalating clinical problem since these biologics are increasingly being used for the treatment of various inflammatory, autoimmune and malignant diseases [37, 38]. The risk of developing reactions to mAbs depends on the humanization of the $\mathrm{mAb}$ (i.e., fully human mAbs are considered less immunogenic than humanized or chimeric mAbs, which contain variable amounts of sequences of mouse origin), the type of Ig elicited (i.e., IgE vs. IgG), the activation of complement, and the presence of adjuvants and excipients [37]. While most $\mathrm{mAb}$ hypersensitivity reactions are due to cytokine release and lack immune specificity (e.g., fever, rigors, chills, headache, chest/back pain, increased blood pressure, gastrointestinal symptoms), immune-specific hypersensitivity reactions may also occur, and these can overlap with non-immune mechanisms leading to complex clinical presentations [39, 40]. It should be noted that reactions due to cytokine release typically occur upon first administration of the mAb and generally wane rapidly with subsequent exposures.

Hypersensitivity reactions to mAbs are classified as immediate (onset within a few hours of infusion) and non-immediate (onset from a few hours to 14 days after infusion). The reactions can be systemic or local (at the injection site). Immediate hypersensitivity reactions, such 
as urticaria, bronchospasm, and multi-organ anaphylaxis, are mediated by IgE (mast cell/basophil activation) or IgG (basophil activation). IgE-mediated reactions to mAbs typically occur after previously well-tolerated exposures because sensitization has to take place before a reaction can develop. However, IgE-mediated reactions have been noted during the very first administration of cetuximab (a chimeric mAb used in the treatment of colorectal, lung, skin, and head and neck cancers) due to pre-existing IgE antibodies directed against an oligosaccharide (i.e., galactose-alpha-1,3-galactose [alpha-gal]) present on this $\mathrm{mAb}[41,42]$. In IgE-mediated reactions, skin tests may be positive and/or tryptase may be elevated at the time of the reaction.

IgG-mediated reactions have been noted upon repeat exposure to infliximab. These reactions resemble IgEmediated reactions, although symptoms may be amplified by complement activation. Skin tests are negative in IgGmediated reactions [43].

The most common manifestation of a non-immediate hypersensitivity reaction to mAbs is a serum sicknesslike reaction with vasculitic manifestations (e.g., fever, malaise, arthralgia/arthritis, jaw pain or tightness, an erythematous or urticarial skin eruption, purpura, and conjunctival hyperemia) that typically appears 5-7 days after the infusion [43]. Maculopapular exanthema is another delayed reaction that has been noted with infliximab and abciximab. Rare, nonimmediate reactions, such as symmetrical drug-related intertriginous and flexural exanthema (SDRIFE), SJS and TEN, have also been attributed to mAbs.

The management of mAb hypersensitivity reactions is still evolving, and evidence regarding the value of skin and intradermal testing is expanding. For some mAbs, these tests have shown positive results, suggesting that reactions were IgE-mediated. When severe hypersensitivity reactions to mAbs occur, an alternate drug should be given whenever possible. For example, panitumumab can replace cetuximab in patients with allergic reactions mediated by IgE antibodies to alphagal [44]. Desensitization is only indicated when the $\mathrm{mAb}$ is considered first-line therapy. When designed appropriately, desensitization protocols have proven successful in addressing both immune- and nonimmune-mediated reactions. In these protocols, the rate of the $\mathrm{mAb}$ infusion is adjusted according to the severity of the initial hypersensitivity event, eventual breakthrough reactions during each desensitization course, and body weight (in pediatric patients) [45]. Desensitization is contraindicated in severe delayed hypersensitivity reactions, including serum sickness-like reactions.
Premedication is an important adjunct to desensitization, and it should be tailored to the clinical characteristics of the index reaction. Depending on these characteristics, premedication may include $\mathrm{H} 1$ or $\mathrm{H} 2$ antihistamines, montelukast, aspirin, acetaminophen, corticosteroids and/or benzodiazepines.

\section{Multiple drug hypersensitivity syndrome}

Multiple drug hypersensitivity (MDH) is a rather novel syndrome which is characterized by delayed hypersensitivity reactions to two or more structurally unrelated drugs [43]. The initial reaction generally presents as severe exanthems or DRESS, and is associated with massive T-cell activation. Significant numbers of lymphoblasts are present in the circulation for weeks to months following this initial reaction. Upon concurrent or subsequent exposure to a non-cross reactive drug, a second specific T-cell sensitization takes place, leading to clinical manifestations which may be similar to or vary from the initial reaction. Subsequent reactions may include exanthema, erythroderma, DRESS (with similar or different organ involvement as the initial DRESS), SIS, TEN, hepatitis, and agranulocytosis. The timing of these subsequent reactions can vary from weeks of the initial reaction, to months or even years after resolution of the initial presentation. The drugs involved in eliciting the MDH syndrome are the same as for DRESS, and they are usually given in rather high doses. Fixed drug combination therapies such as TMP-SMX or piperacillin/ tazobactam are frequently involved in MDH [43].

To lower the risk of developing MDH in patients with severe T-cell-mediated reactions, experts have recommended the following strategies: (1) minimizing the use of further drugs; (2) omitting antipyretics; (3) avoiding antibiotics unless they are absolutely indicated; (4) if needed, choosing drugs that can be given at a lower dose (e.g., $<50 \mathrm{mg} /$ day); (5) dampening the hyperactivation of the immune system with corticosteroid therapy; and (6) attempting to create a therapy-free interval for days to weeks [43].

\section{De-labelling of medication allergies}

As discussed earlier, penicillin allergy is a common drug allergy diagnosis. However, studies have shown that among patients who report a penicillin allergy, more than $80 \%$ have a negative response to penicillin skin testing [46]. Furthermore, $90 \%$ of adult inpatients tolerate penicillin upon further evaluation [47].

Patients with a suspected allergy to penicillin are often not referred to an allergist for evaluation and are instead prescribed alternate antimicrobials that may be less effective, more toxic or more expensive. In fact, a penicillin allergy label has been associated with negative 
clinical and administrative outcomes, including more hospitalizations, increased antibiotic-resistant infections and greater medical costs. As a result, there has been increased focus within North America to remove the label of 'drug allergy', particularly to penicillin [48-51]. These programs are multidisciplinary in nature, with involvement of antimicrobial stewardship groups, allergists and pharmacists, and have been shown to improve patient-related outcomes and reduce health care costs. With increasing evidence that penicillin de-labelling initiatives are successful, it will become increasingly important to consider de-labelling other medications as part of these initiatives.

\section{Prevention of future reactions}

Prevention of future reactions is an essential part of patient management. The patient should be provided with written information about which drugs to avoid (including over-the-counter medications). The drugs should be highlighted in the hospital notes and within electronic records (where available), and the patient's family physician should be informed of the drug allergy. Engraved allergy bracelets/necklaces, such as those provided by MedicAlert ${ }^{\circledR}$, should also be considered, particularly if the patient has a history of severe druginduced allergic reactions [16].

\section{Conclusions}

Drug allergy is a common clinical problem; assessment by an allergist is important for appropriate diagnosis and management of the condition. Diagnosis relies on a careful history and physical examination and, in some instances, skin testing and graded challenges may be required. The mainstay of treatment for drug allergy is avoidance of the offending drug. When available, alternative medications with unrelated chemical structures should be substituted. Cross-reactivity among drugs should be taken into consideration when choosing alternative medications. If a particular drug to which the patient is allergic is indicated and there is no suitable alternative, induction of drug tolerance procedures may be considered to induce temporary tolerance to the drug.

\section{Key take-home messages}

- Drug allergy encompasses a spectrum of immunologically mediated hypersensitivity reactions with varying mechanisms and clinical presentations.

- Risk factors for drug allergy include age (more common in young/middle-aged adults), gender (more common in females), genetic polymorphisms, certain viral infections (HIV and herpes viruses) and drug-related factors (topical and IV/intramuscular routes of administration are more immunogenic than oral administration).

- Referral to an allergist is important for appropriate diagnosis and treatment of drug allergy.

- Diagnosis requires a thorough drug history, including dates of administration, drug formulation, dosage and route of administration, as well as clinical symptoms and their timing and duration in relation to drug exposure; skin testing and graded challenges may also be required.

- The skin is the organ most frequently affected by drug-induced allergic reactions, however, many other organ systems may be involved, including multi-organ reactions such as anaphylaxis.

- The mainstay of treatment is avoidance of the offending drug; alternative medications with unrelated chemical structures should be substituted when possible.

- If a particular drug to which the patient is allergic is indicated, induction of drug tolerance procedures may be considered to induce temporary tolerance to the drug.

\section{Abbreviations}

ADR: adverse drug reaction; ASA: acetylsalicylic acid; NSAIDs: non-steroidal anti-inflammatory drugs; mAbs: monoclonal antibodies; IgE: immunoglobulin E; IgG: immunoglobulin G; IgM: immunoglobulin M; APCs: antigen-presenting cells; ACE: angiotensin-converting enzyme; HLA: human leukocyte antigen; HIV: human immunodeficiency virus; EBV: Epstein-Barr virus; SJS: StevensJohnson syndrome; TEN: toxic epidermal necrolysis; DRESS: drug rash with eosinophilia and systemic symptoms; DILE: drug-induced lupus erythematosus; SPT: skin prick testing; IV: intravenous; TMP-SMX: trimethoprim-sulfamethoxazole; RCM: radiocontrast media; COX-1: cyclooxygenase-1; COX-2: cyclooxygenase-2; SDRIFE: symmetrical drug-related intertriginous and flexural exanthema; MDH: multiple drug hypersensitivity; alpha-gal: galactosealpha-1,3-galactose; MHC: major histocompatibility complex.

\section{Declarations}

Authors' contributions All authors wrote and/or edited sections of the manuscript. All authors read and approved the final manuscript.

\section{Author details}

${ }^{1}$ University of Manitoba, Winnipeg, MB, Canada. ${ }^{2}$ McGill University, Montreal, QC, Canada. ${ }^{3}$ University of British Columbia, Vancouver, BC, Canada.

\section{Acknowledgements}

This article is an update to the Drug Allergy article that originally appeared in the supplement entitled, Practical Guide to Allergy and Immunology in Canada, which was published in Allergy, Asthma \& Clinical Immunology in 2011 (available at: https://aacijournal.biomedcentral.com/articles/supplements/volum e-7-supplement-1).

The authors would like to thank Julie Tasso for her editorial services and assistance in the preparation of this manuscript.

\section{Competing interests}

Dr. Richard Warrington is the past president of the Canadian Society of Allergy and Clinical Immunology and Editor-in-Chief of Allergy, Asthma \& Clinical 
Immunology. He has received consulting fees and honoraria from Nycomed, CSL Behring, Talecris, Grifols, Novartis and Shire.

Dr. Tiffany Wong and Dr. Fanny Silviu-Dan have no competing interests to disclose.

\section{Availability of data and materials}

Data sharing not applicable to this article as no datasets were generated or analyzed during the development of this review.

\section{Consent for publication}

Not applicable.

\section{Ethics approval and consent to participate}

Ethics approval and consent to participate are not applicable to this review article.

\section{Funding}

Publication of this supplement has been supported by AstraZeneca, Boehringer Ingelheim, CSL Behring Canada Inc., MEDA Pharmaceuticals Ltd., Merck Canada Inc., Pfizer Canada Inc., Shire Pharma Canada ULC, Stallergenes Greer Canada, Takeda Canada, Teva Canada Innovation, Aralez Tribute and Pediapharm

\section{About this supplement}

This article has been published as part of Allergy, Asthma \& Clinical Immunology Volume 14 Supplement 2, 2018: Practical guide for allergy and immunology in Canada 2018. The full contents of the supplement are available online at https ://aacijournal.biomedcentral.com/articles/supplements/volume-14-suppl ement-2.

\section{Publisher's Note}

Springer Nature remains neutral with regard to jurisdictional claims in published maps and institutional affiliations.

\section{Published: 12 September 2018}

\section{References}

1. Khan DA, Solensky R. Drug allergy. J Allergy Clin Immunol. 2010;125:S126-37.

2. Lazarou J, Pomeranz BH, Corey PN. Incidence of adverse drug reactions in hospitalized patients: a meta-analysis of prospective studies. JAMA. 1998:279(15):1200-5.

3. Gandhi TK, Weingart SN, Borus J, Seger AC, Peterson J, Burdick E, Seger DL, Shu K, Federico F, Leape LL, Bates DW. Adverse drug events in ambulatory care. N Engl J Med. 2003;348(16):1556-64.

4. Vervloet D, Durham S. Adverse reactions to drugs. BMJ. 1998;316(7143):1511-4.

5. Sylvia LM. Drug allergy, pseudoallergy and cutaneous diseases. In: Tisdale JE, Miller DA, editors. Drug-induced diseases: prevention, detection, and management. 2nd ed. Bethesda: American Society of Health-System Pharmacists; 2010.

6. Riedl MA, Castillas AM. Adverse drug reactions: types and treatment options. Am Fam Physician. 2003:68(9):1781-90.

7. Gell PGH, Coombs RRA. Clinical aspects of immunology. 3rd ed. Oxford: Blackwell Scientific Publications; 1975.

8. Pichler WJ. Delayed drug hypersensitivity reactions. Ann Intern Med. 2003;139(8):683-93.

9. Posadas SJ, Pichler WJ. Delayed drug hypersensitivity reactions: new concepts. Clin Exp Allergy. 2007;37(7):989-99.

10. Yun J, Cai F, Lee FJ, Pichler WJ. T-cell-mediated drug hypersensitivity: immune mechanisms and their clinical relevance. Asia Pac Allergy. 2016;6(2):77-89.

11. Friedmann PS, Ardern-Jones M. Patch testing in drug allergy. Curr Opin Allergy Clin Immunol. 2010;10(4):291-6.

12. Schnyder B. Approach to the patient with drug allergy. Immunol Allergy Clin N Am. 2009;29(3):405-18.
13. Barranco P, Lopez-Serrano MC. General and epidemiological aspects of allergic drug reactions. Clin Exp Allergy. 1998;28(Suppl 4):61-2.

14. Adkinson NF Jr. Risk factors for drug allergy. J Allergy Clin Immunol. 1984;74(4 Pt 2):567-72.

15. Pirmohamed $M$, Park BK. Adverse drug reactions: back to the future. Br J Clin Pharmacol. 2003;55(5):486-92.

16. Mirakian R, Ewan PW, Durham SR, Youlten LJ, Dugué $P$, Friedmann PS, English JS, Huber PA, Nasser SM, BSACI. BSACI guidelines for the management of drug allergy. Clin Exp Allergy. 2009;39(1):43-61.

17. Caubet JC, Kaiser L, Lemaitre B, Fellay B, Gervaix A, Eigenmann PA. The role of penicillin in benign skin rashes in childhood: a prospective study based on drug rechallenge. J Allergy Clin Immunol. 2011;127(1):218-22.

18. Rubio M, Bousquet PJ, Gomes E, Romano A, Demoly P. Results of drug hypersensitivity evaluations in a large group of children and adults. Clin Exp Allergy. 2012;42(1):123-30.

19. Barbaud A. Drug patch testing in systemic cutaneous drug allergy. Toxicology. 2005;209(2):209-16.

20. Sanz ML, Gamboa PM, De Weck AL. Cellular tests in the diagnosis of drug hypersensitivity. Curr Pharm Des. 2008;14(27):2803-8.

21. Hausmann OV, Gentinetta T, Bridts CH, Ebo DG. The basophil activation test in immediate-type drug allergy. Immunol Allergy Clin North Am. 2009;29(3):555-66.

22. Aberer W, Kränke B. Provocation tests in drug hypersensitivity. Immunol Allergy Clin North Am. 2009;29(3):567-84.

23. Atanasković-Marković M, Gaeta F, Gavrović-Jankulović M, Velicković $\mathrm{TC}$, Valluzzi RL, Romano A. Tolerability of imipenem in children with IgE-mediated hypersensitivity to penicillins. J Allergy Clin Immunol. 2009;124(1):167-9.

24. Frumin J, Gallagher JC. Allergic cross-sensitivity between penicillin, carbapenem, and monobactam antibiotics: what are the chances? Ann Pharmacother. 2009:43(2):304-15.

25. Saxon A, Adelman DC, Patel A, Hajdu R, Calandra GB. Imipenem cross-reactivity with penicillin in humans. J Allergy Clin Immunol. 1988;82(2):213-7

26. Saxon A, Hassner A, Swabb EA, Wheeler B, Adkinson NF Jr. Lack of crossreactivity between aztreonam, a monobactam antibiotic, and penicillin in penicillin-allergic subjects. J Infect Dis. 1984;149(1):16-22.

27. Adkinson NF Jr. Immunogenicity and cross-allergenicity of aztreonam. Am J Med. 1990;88(3C):12S-5S.

28. Kelkar PS, Li JT. Cephalosporin allergy. N Engl J Med. 2001;345(11):804-49.

29. Romano A, Guéant-Rodriguez RM, Viola M, Pettinato R, Guéant JL. Crossreactivity and tolerability of cephalosporins in patients with immediate hypersensitivity to penicillins. Ann Intern Med. 2004;141(1):16-22.

30. Macy E, Ngor EW. Safely diagnosing clinically significant penicillin allergy using only penicilloyl-poly-lysine, penicillin, and oral amoxicillin. J Allergy Clin Immunol Pract. 2013;1(3):258-63.

31. Romano A, Gaeta F, Valluzzi RL, Caruso C, Rumi G, Bousquet PJ. IgE-mediated hypersensitivity to cephalosporins: cross-reactivity and tolerability of penicillins, monobactams, and carbapenems. J Allergy Clin Immunol. 2010;126(5):994-9.

32. Zawodniak A, Lochmatter P, Beeler A, Pichler WJ. Cross-reactivity in drug hypersensitivity reactions to sulfasalazine and sulfamethoxazole. Int Arch Allergy Immunol. 2010;153(2):152-6.

33. Strom BL, Schinnar R, Apter AJ, Margolis DJ, Lautenbach E, Hennessy S, Bilker WB, Pettitt D. Absence of cross-reactivity between sulfonamide antibiotics and sulfonamide nonantibiotics. N Engl J Med. 2003;349(17):1628-35.

34. Dibbern DA, Montanaro A. Allergies to sulfonamide antibiotics and sulfurcontaining drugs. Ann Allergy Asthma Immunol. 2008;100(2):91-100.

35. Gurrieri C, Weingarten TN, Martin DP, Babovic N, Narr BJ, Sprung J, Volcheck GW. Allergic reactions during anesthesia at a large United States referral center. Anesth Analg. 2011;113(5):1202-12.

36. Ewan PW, Dugué $P$, Mirakian R, Dixon TA, Harper JN, Nasser SM, BSACl. $\mathrm{BSACl}$ guidelines for the investigation of suspected anaphylaxis during general anaesthesia. Clin Exp Allergy. 2010;40(1):15-31.

37. Picard M, Galvão VR. Current knowledge and management of hypersensitivity reactions to monoclonal antibodies. J Allergy Clin Immunol Pract. 2017;5(3):600-9.

38. Vultaggio A, Castells MC. Hypersensitivity reactions to biologic agents. Immunol Allergy Clin North Am. 2014;34(3):615-32. 
39. Lenz HJ. Management and preparedness for infusion and hypersensitivity reactions. Oncologist. 2007;12(5):601-9.

40. Patel SV, Khan DA. Adverse reactions to biologic therapy. Immunol Allergy Clin North Am. 2017;37(2):397-412.

41. Chung CH, Mirakhur B, Chan E, Le QT, Berlin J, Morse M, Murphy BA, Satinover SM, Hosen J, Mauro D, Slebos RJ, Zhou Q, Gold D, Hatley T, Hicklin DJ, Platts-Mills TA. Cetuximab-induced anaphylaxis and lgE specific for galactose-alpha-1,3-galactose. N Engl J Med. 2008;358(11):1 109-17.

42. Hausmann OV, Seitz M, Villiger PM, Pichler WJ. The complex clinical picture of side effects to biologicals. Med Clin North Am. 2010;94(4):791-804.

43. Pichler WJ, Srinoulprasert Y, Yun J, Hausmann O. Multiple drug hypersensitivity. Int Arch Allergy Immunol. 2017;172(3):129-38.

44. Langerak A, River G, Mitchell E, Cheema P, Shing M. Panitumumab monotherapy in patients with metastatic colorectal cancer and cetuximab infusion reactions: a series of four case reports. Clin Colorectal Cancer. 2009;8(1):49-54.

45. Sloane D, Govindarajulu U, Harrow-Mortelliti J, Barry W, Hsu FI, Hong D, Laidlaw T, Palis R, Legere H, Bunyavanich S, Breslow R, Wesemann D, Barrett N, Brennan P, Chong HJ, Liu A, Fernandez J, Fanning L, Kyin T, Cahill K, Bankova L, Lynch A, Berlin S, Campos S, Fuchs C, Mayer R, Matulonis U, Castells M. Safety, costs, and efficacy of rapid drug desensitizations to chemotherapy and monoclonal antibodies. J Allergy Clin Immunol Pract. 2016;4(3):497-504.
46. Albin S, Agarwal S. Prevalence and characteristics of reported penicillin allergy in an urban outpatient adult population. Allergy Asthma Proc. 2014;35(6):489-94.

47. Joint Task Force on Practice Parameters, American Academy of Allergy, Asthma and Immunology, American College of Allergy, Asthma and Immunology, Joint Council of Allergy, Asthma and Immunology. Drug allergy: an updated practice parameter. Ann Allergy Asthma Immunol. 2010;105(4):259-73.

48. Rimawi RH, Cook PP, Gooch M, Kabchi B, Ashraf MS, Rimawi BH, Gebregziabher M, Siraj DS. The impact of penicillin skin testing on clinical practice and antimicrobial stewardship. J Hosp Med. 2013;8(6):341-5.

49. Picard M, Paradis L, Nguyen M, Bégin P, Paradis J, Des Roches A. Outpatient penicillin use after negative skin testing and drug challenge in a pediatric population. Allergy Asthma Proc. 2012;33(2):160-4.

50. Bourke J, Hollingsworth PR, McLean-Tooke P, et al. Penicillin de-labelling in tertiary care clinics: safe and efficacious but incomplete effectiveness. Int Med J. 2012;42(Supplement 4):21-2.

51. Trubiano J, Phillips E. Antimicrobial stewardship's new weapon? A review of antibiotic allergy and pathways to 'de-labeling'. Curr Opin Infect Dis. 2013;26(6):526-37.
Ready to submit your research? Choose BMC and benefit from:

- fast, convenient online submission

- thorough peer review by experienced researchers in your field

- rapid publication on acceptance

- support for research data, including large and complex data types

- gold Open Access which fosters wider collaboration and increased citations

- maximum visibility for your research: over 100M website views per year

At BMC, research is always in progress.

Learn more biomedcentral.com/submissions 